\title{
Liquid film spray deposition of transparent conducting oxide coatings
}

\author{
J. Puetz*, G. Gasparro, M.A. Aegerter \\ Institut für Neue Materialien, Coating Technology, D-66123 Saarbruecken, Germany
}

\begin{abstract}
A room temperature spraying technique was developed for the deposition of transparent conducting sol-gel ATO (antimony doped tin oxide, $\mathrm{SnO}_{2}: \mathrm{Sb}$ ) coatings, based on an $0.2 \mathrm{M}$ solution of $\mathrm{SnCl}_{2}$ in 1-propanol with $5 \mathrm{~mol} . \% \mathrm{SbCl}_{3}$ and a mixture of 2isopropoxyethanol and 2-butoxyethanol as additives with a low evaporation rate. The selection of solvents and the adjustment of the evaporation rate are crucial to obtain uniform films and to avoid film defects like wetting problems or an orange peel effect. The ATO coatings were spray deposited manually on complex shaped substrates as well as on large area flat substrates with a size up to $21 \times 30 \mathrm{~cm}^{2}$ by means of an industrial flat spray coater. The resulting ATO coatings are characterised by a thickness of up to $120 \mathrm{~nm}$, a resistivity of approximately $2 \times 10^{-2} \Omega \mathrm{cm}$ and a transmission $T>85 \%$ in the visible, which is similar to the properties obtained for dip coated films.
\end{abstract}

Keywords: Sol-gel process; Electrical properties and measurements; Optical coatings; Tin oxide

\section{Introduction}

Transparent conducting oxide coatings are important components in a large number of applications, due to the unique combination of an electrical conductivity with a high optical transmission in the visible. Typical fields of applications for such coatings are, e.g. the use as electrodes in displays, solar cells and heating elements or in the provision of electromagnetic shielding or antistatic properties $[1,2]$.

The most commonly used materials in these fields are tin doped indium oxide (ITO, $\mathrm{In}_{2} \mathrm{O}_{3}: \mathrm{Sn}$ ), fluorine doped tin oxide $\left(\mathrm{FTO}, \mathrm{SnO}_{2}: \mathrm{F}\right)$, antimony doped tin oxide (ATO, $\mathrm{SnO}_{2}: \mathrm{Sb}$ ) and aluminium doped zinc oxide (AZO, $\mathrm{ZnO}: \mathrm{Al}$ ). The corresponding coatings are usually applied by physical or chemical vapour deposition (PVD, CVD) as these techniques result in the lowest sheet resistance and guarantee good optical properties [1]. In the case of FTO, a hot spraying process (spray pyrolysis) has been established, where the coating solution is directly sprayed on the hot substrate at the glass manufacturer (high-throughput hot-end coating), mainly for the use as low-E (low emissivity) glasses in architecture [1]. However, these technologies are rather costly and optical quality coatings can only be deposited on flat or weakly bent substrates.

*Corresponding author.
For the deposition of TCO coatings it is, therefore, desirable to also have access to the ease and flexibility of liquid film coating techniques [3]. In this way, a large variety of wet deposition techniques like dip, spin and roll coating or spraying can be used to coat almost all substrate shapes. For sol-gel coatings, the most popular deposition techniques up to now is the dip coating process, as it is easy to perform and results in high quality thin films. However, compared to the dip coating process, the spraying technique exhibits some fundamental advantages if extreme thickness uniformity is not required:

- higher speed of deposition,

- higher flexibility in the shape of the substrate,

- lower total amount of coating solution,

- reduced aging and contamination of the coating solution,

- availability of the spraying equipment.

The feasibility of the spraying of sol-gel coatings has already been shown for $\mathrm{PbO}-\mathrm{SiO}_{2}$ coatings containing $\mathrm{Au}$ colloids [4], as well as for $\mathrm{SiO}_{2}, \mathrm{TiO}_{2}, \mathrm{ATO}$ and ITO $[5,6]$. In all these cases, an approach from paint and lacquer engineering based on the use of high boiling point solvents [7,8] like glycols or glycol ethers were used to obtain homogeneous films. 


\section{Experimental}

Borosilicate glass substrates (borofloat 33, Schott) with a size of $10 \times 10 \mathrm{~cm}^{2}$ and $21 \times 30 \mathrm{~cm}^{2}$ were polished manually with $\mathrm{CeO}_{2}$ powder and subsequently cleaned with an industrial dish washer using an alkaline detergent and an acidic neutralizer. A $0.2 \mathrm{M}$ solution of $\mathrm{SnCl}_{2}$ (Fluka, 98\%) in 1-propanol with $5 \mathrm{~mol} \% \mathrm{SbCl}_{3}$ (Fluka, 99\%), 20 vol.\% 2-isopropoxyethanol and 10 vol.\% 2-butoxyethanol was prepared and filtered with a $0.2 \mu \mathrm{m}$ syringe filter. This coating solution was spray deposited manually by means of a spray gun (SATA Minijet, $0.5 \mathrm{~mm}$ nozzle) followed by a drying step at $250{ }^{\circ} \mathrm{C}(15 \mathrm{~min})$ and a subsequent heating at 500 to $550{ }^{\circ} \mathrm{C}$ for $15 \mathrm{~min}$ in air. Additionally, large area substrates were coated by means of an industrial flat spray coater (Venjakob, DSG-Single) with two high volume low pressure spray guns (Krautzberger HVLP) mounted crosswise on one axis. A mist control during spraying was obtained with both a water curtain and a flux of filtered air $\left(7000 \mathrm{~m}^{3} / \mathrm{h}\right)$. The thus deposited coatings were heat treated in-line after passing a flashoff zone by means of an IR-heated zone (approx. 200 ${ }^{\circ} \mathrm{C}$ ) and a continuous furnace with a temperature of 550 ${ }^{\circ} \mathrm{C}$.

The thickness $d$ of the ATO coatings was determined by profilometry (Tencor P10) after etching with $\mathrm{HCl}$ and $\mathrm{Zn}$ dust. With the same instrument, the average roughness $R_{\mathrm{a}}$ was obtained from a measurement over a distance of $0.5 \mathrm{~mm}$. The sheet resistance $R_{\square}$ was measured by the 4-point-technique using Au tips in a linear arrangement (34401 A, Hewlett-Packard). The optical transmission $T$ of the samples was determined under normal incidence in the spectral range between 200 and $3000 \mathrm{~nm}$ against air as a reference (Cary 5E, Varian).

\section{Results and discussion}

\subsection{Flat substrates}

Coating solutions for sol-gel dip coating are usually based on low boiling point solvents like ethanol or 2propanol in order to guarantee a fast and complete solvent evaporation. If such solutions are used for a spray deposition, the resulting coating quality is by far not satisfying. As most of the solvent already evaporates during the flight stage, an almost dry powder hits the substrates and the film mobility is not sufficient anymore to level out and form a homogeneous film.

The problem can easily be overcome by the addition of solvents with lower evaporation rates, which is a basic concept for the control of film formation of paints and lacquers. The approach is also suitable for the much thinner sol-gel films as the early processes of film formation are similar in both cases. The evaporation rate depends on a variety of parameters and environmental conditions [8], so that an empirical treatment is more or less unavoidable. A good approximation for the evaporation rate, however, is given by the boiling point of the solvent as its volatility generally decreases with increasing boiling point within a group of chemically related solvents. However, besides the evaporation rate, solvent properties like the substrate wetting and interactions with the sol-gel precursors are also decisive so that mainly glycols and glycol ethers are used as additives.

Typical coating defects that come from a mismatch of solvents are shown in Fig. 1 together with a homogeneous, defect-free coating. The already mentioned wetting problems on glass substrates (Fig. 1b) were especially observed with glycols (e.g. ethylene glycol) but also for high contents of the less polar 2-butoxyethanol, so that preferably the short-chained homologues were chosen. One of the most frequent and obstinate defects is the so-called orange peel appearance shown in Fig. 1c. It origins from the selective evaporation of the solvent, which in consequence leads to local disturbances of the surface tension known as the Marangoni effect [3]. As the resulting thickness variations are particularly visible in the case of highly refractive materials like tin oxide, a careful adaptation of the solvent mixture is necessary to obtain coatings in optical quality. Fig. 1d shows a hazy, powdery film, obtained when the evaporation rate is too high and in consequence, the solution is already dried when it hits the substrate surface.

For the given sol-gel ATO precursor system, homogeneous, uniform coatings (Fig. 1a) were obtained with 1-propanol as the main solvent and the addition of 20 vol.\% of 2-isopropoxyethanol and 10 vol.\% 2-butoxyethanol. The obtained solution can easily be sprayed and shows only a low sensitivity to humidity (40 to $70 \% \mathrm{RH})$ and temperature changes which is otherwise a critical point of sol-gel processing.

The properties of the obtained ATO coatings are similar to those found for dip coated samples made from the same precursor system [9]. Coatings with a thickness $d$ of up to 110 to $120 \mathrm{~nm}$ and an average roughness $R_{\mathrm{a}}$ of less than $1 \mathrm{~nm}$ can be deposited, whereas thicker coatings, like in the case of dip coated films, show cracks and, therefore, exhibit a poor electrical conductivity. The sheet resistance $R_{\square}$ of the sprayed sol-gel ATO coatings is approximately 2 to $4 \mathrm{k} \Omega_{\square}$, which corresponds to a resistivity of approximately $2 \times 10^{-2}$ $\Omega \mathrm{cm}$. This is the same resistivity that is obtained in dip coated sol-gel ATO single layers. Accordingly, the transmission spectrum of the obtained ATO coating (Fig. 2) is similar to that of dip coated films with a transmission $T>85 \%$ in the visible.

For the coating of large area flat glass substrates, a continuous flat spray coater with an integrated drying 

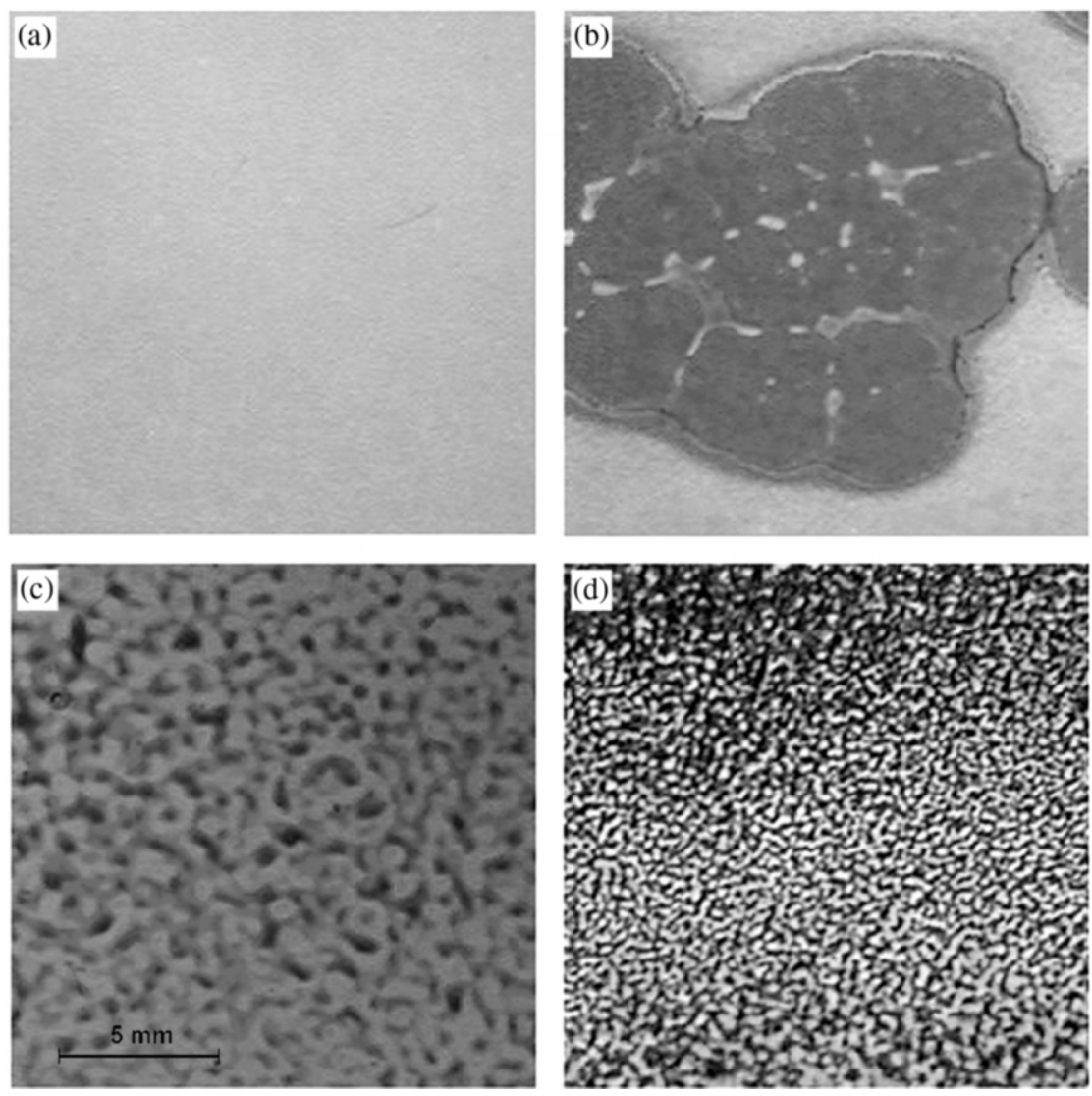

Fig. 1. Appearance (in reflection) and typical defects of sprayed sol-gel ATO coatings on borosilicate glass substrates. (a) Homogeneous defectfree coating, (b) wetting problems, (c) orange peel appearance, (d) drying of the solution during the flight stage.

and heating zone was used. The resulting coatings deposited on a $21 \times 30 \mathrm{~cm}^{2}$ substrate had a mean thickness of $(90 \pm 10) \mathrm{nm}$ and a sheet resistance distribution as shown in Fig. 3. The coating still shows a gradient in the sheet resistance, which is largely caused

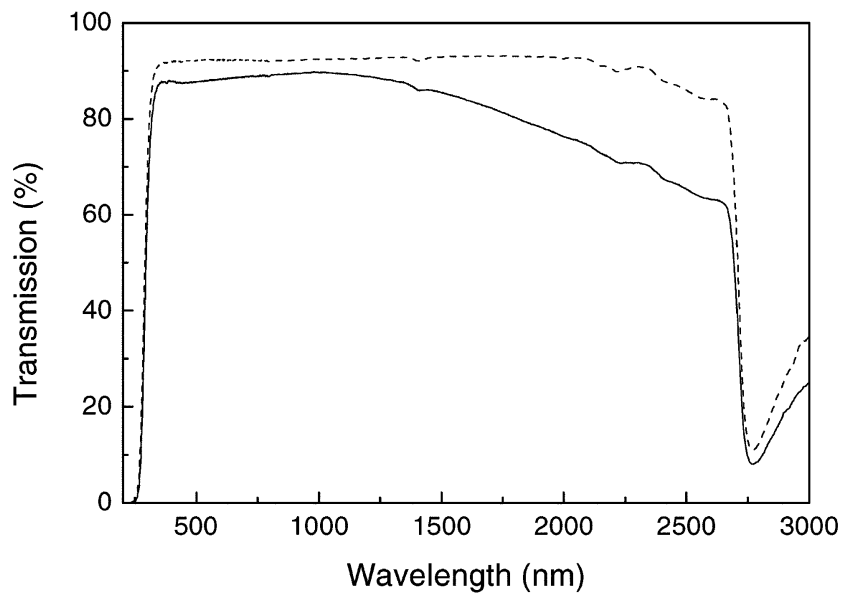

Fig. 2. Optical transmission spectra of a spray deposited sol-gel ATO sample $\left(d \approx 80 \mathrm{~nm}, R_{\square} \approx 4 \mathrm{k} \Omega_{\square}\right)$ (solid) and the borosilicate glass substrate (dashed). by a gradient in the thickness. The reason for this inhomogeneity is to be found in the heating, rather than in the spray deposition process, so that after a further optimisation thickness and sheet resistance deviations of $\pm 5 \mathrm{~nm}$ and $\pm 2 \mathrm{k} \Omega_{\square}$, respectively, should be realizable,

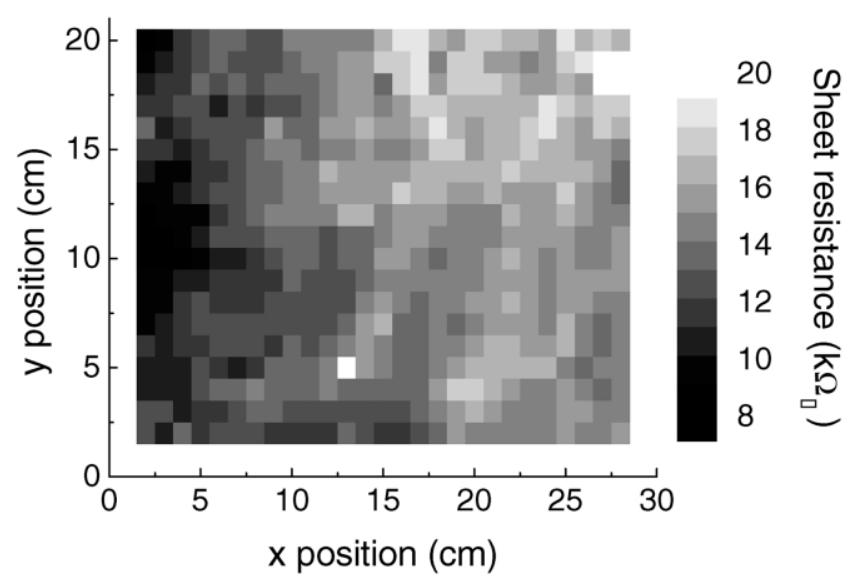

Fig. 3. Distribution of the sheet resistance $R_{\square}$ of a sol-gel ATO coating $(d \approx 90 \mathrm{~nm})$ deposited with an industrial flat spray coater on a $21 \times 30 \mathrm{~cm}^{2}$ borosilicate glass substrate. 


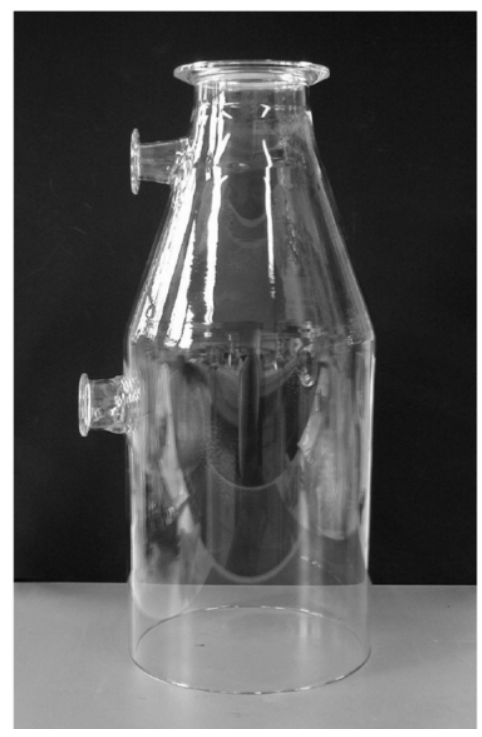

Fig. 4. Spray deposited antistatic sol-gel ATO coating (3 to $5 \mathrm{k} \Omega$ ) on the outer side of a borosilicate glass tube with flange fittings (46 $\mathrm{cm}, \varnothing 22 \mathrm{~cm}$ ).

as indicated by the lower vertical variations. For the discussion of the results, it should be kept in mind that the electrical properties are very sensitive to any kind of change in the morphology and the composition of the coating.

\subsection{Complex-shaped substrates}

A major advantage of the spraying technique over other wet coating techniques is the possibility to coat substrates with complex shapes. Such an ATO coating was successfully applied to a large glass tube with flanges used in chemical or food technology (Fig. 4), to realise antistatic properties. Even with a manual spraying technique, a homogeneous, transparent coating with an average sheet resistance of 3 to $5 \mathrm{k} \Omega_{\square}$ and a resistance of $35 \mathrm{k} \Omega$ over the total length was obtained. Due to the high flexibility of the spraying technique, also the lateral flange fittings could be provided with antistatic properties without loss in transparency. In addition to the electrical conductivity, the applied tin oxide coating acts as a protective coating due to its high chemical and mechanical resistance.

\section{Conclusions}

It was shown for transparent conducting ATO that sol-gel coatings could easily be deposited defect-free and optically flawless by the spraying technique. This was achieved by a modification of the coating solution by addition of glycol ethers with a lower evaporation rate. The substrates thus can be coated comfortably at room temperature and can then be treated afterwards in separate drying and heating steps. The properties of the resulting ATO coatings are similar to those obtained by sol-gel dip coating with a thickness of up to $120 \mathrm{~nm}$ and a resistivity of approximately $2 \times 10^{-2} \Omega \mathrm{cm}$. The presented spray coating technique allows a fast and economic deposition on large area flat substrates and complex shaped substrates as demonstrated both by manual spraying and by means of an industrial flat spray coater. The thus prepared ATO coatings can be used to give excellent antistatic properties to a variety of glass substrates like, e.g. preformed parts or viewing windows.

\section{References}

[1] H.J. Gläser, Large Area Glass Coating, Von Ardenne Anlagentechnik GmbH, Dresden, 2000.

[2] B.G. Lewis, D.C. Paine, MRS Bulletin, 25 (2000) 22.

[3] S.F. Kistler, P.M. Schweizer, Liquid Film Coating, Chapman and Hall, London, 1997.

[4] C. Fink-Straube, A. Kalleder, T. Koch, M. Mennig, DE Patent No. 19840525 A1, 6 Sept. 1998.

[5] C. Löser, C. Rüssel, Glass Sci. Technol. (Glasstech. Ber.) 73 (2000) 270.

[6] C. Löser, C. Rüssel, Glass Sci. Technol. (Glasstech. Ber.) 74 (2001) 106.

[7] J. Bieleman, Additives For Coatings, Wiley, Weinheim, 2001.

[8] D. Stoye, Paints, Coatings and Solvents, Wiley-VCH, Weinheim, 1993.

[9] G. Gasparro, J. Puetz, unpublished. 